\title{
Electrospun PLLA Membranes for Caffeine Delivery: Diffusional Approach
}

\author{
Ana Paula S. Immich ${ }^{1}$, José Antonio Torneroํ, Francesc Cano Casas ${ }^{1}$, Manuel J. Lis Arias ${ }^{2}$ \\ ${ }^{1}$ Institut d'Investigació Tèxtil i Cooperació Industrial de Terrassa, Universitat Politècnica de Catalunya, Terrassa, \\ Spain; ${ }^{2}$ Chemical Engineering Department, Universitat Politècnica de Catalunya, Terrassa, Spain
}

Correspondence to: Manuel J. Lis Arias, Manuel-jose.lis@upc.edu

Keywords: Diffusion, Polymer, Nano Escale, Kinetics

Received: November 8, 2017 Accepted: December 26, $2017 \quad$ Published: December 29, 2017

Copyright $\odot 2017$ by authors and Scientific Research Publishing Inc.

This work is licensed under the Creative Commons Attribution-NonCommercial International License (CC BY-NC 4.0).

http://creativecommons.org/licenses/by-nc/4.0/

\section{(c) (1) (9) Open Access}

\section{ABSTRACT}

One of the great advantages of electrospun fibers is the large tridimensional area produced, capable of storing any type of material. The aim of our investigation is to study the electrospinning technique to produce polymer membranes for drug delivery applications, given their large surface area and ability to transport a bioactive compound. A mathematical modeling of the delivery system kinetics was also studied to find the mechanism that controls the releasing process. Results showed that electrospinning could provide regular and smooth membranes suitable for drug delivery processes. The mathematical modeling also proved that thicker PLLA membranes exhibited a Fickian diffusion behavior during the drug transport, presenting better control in drug delivery processes.

\section{INTRODUCTION}

For drug delivery systems based on membrane devices, the morphology of membrane fibers is extremely important for drug release performance. When the diameters of polymer fiber materials are shrunk from micrometers (e.g. $10-100 \mu \mathrm{m}$ ) to submicrons or nanometers (e.g. $10 \times 10^{-3}-100 \times 10^{-3} \mu \mathrm{m}$ ), several amazing characteristics appear such as a very large surface area to volume ratio, flexibility in surface functionalities, and superior mechanical performance (e.g. stiffness and tensile strength) compared to any other known form of the material $[1,2]$. These outstanding properties make polymer nanofibers optimal candidates for drug delivery devices. Various drug delivery systems (DDS) have been developed to tailor drug release kinetics and improve the delivery efficiencies according to the actual physiological needs $[3,4]$.

A number of processing techniques such as surface deacetylation [5], self-assembly [6,7] and electrospinning $[8,9]$ have been used to prepare polymer nanofibers in recent years. Electrospinning is a highly versatile technique that combines the use of two techniques, namely electrospray and spinning [10]. 
In the electrospinning process, a polymer solution held by its surface tension at the end of a capillary tube is subjected to an electric field and an electric charge is induced on the liquid surface due to this electric field. When the electric field applied reaches a critical value, the repulsive electrical forces overcome the surface tension forces. Eventually, a charged jet of solution is ejected from the tip of the Taylor cone and an unstable and rapid whipping of the jet occurs in the space between the capillary tip and collector which leads to solvent evaporation, leaving a polymer behind $[11,12]$.

The electrospinning process is controlled by a number of parameters, usually classified as: solution, processing and environmental parameters. Solution parameters include viscosity, conductivity, molecular mass and surface tension. Processing parameters include the electric field applied, distance between capillary tube and collector and solution flow rate. Each of these parameters has a significant influence on the morphology of fibers obtained by electrospinning, and through proper manipulation of these parameters it may possible produce nanofibers with desirable morphology and diameter $[8,13]$. The environmental parameters include relative humidity and room temperature, which also play an important role in the morphology and diameter of electrospun fibers [14].

Electrospun nanofibers are usually used in biomedical applications, such as tissue engineering scaffolds, drug delivery systems, filtration, affinity membranes, biotechnology, environment engineering, energy storage and many other ongoing research studies [15-17]. Among the various potential applications, drug delivery is one of the most promising uses. The high loading capacity, high encapsulation efficiency, simultaneous delivery of diverse therapies, ease operation, cost-effectiveness are appealing features for electrospinning used in drug delivery $[18,19]$. The use of electrospun fiber as drug carriers will be promising in future biomedical applications, especially postoperative local chemotherapy [20,21].

Different systems were developed to control drug delivery. Within these systems, the drug can be loaded in high capacities drug carriers, such as, liposomes, or it can be trapped in degradable polymers to substantial drug release at the desirable site [22]. In drug delivery systems controlled by the polymer, the active molecule is continuously released in therapeutic levels by polymer degradation and diffusion through its pores. However, a number of factors might be considered for the approval of these drug delivery systems, such as, biocompatibility, cytotoxicity, efficiency, cost, etc. Only after analysis of these issues, a drug delivery system can be therapeutically accepted [23]. Poly(L-lactic acid) (PLLA) is one of the most promising biodegradable polymers to be used in drug delivery systems due to its thermoplastic processability, mechanical and biological properties, such as biocompatibility and biodegradability.

The purpose of our study is to develop electrospun membranes from polymeric biomaterials for drug delivery applications. Our research intends to find the best conditions to produce electrospun membranes suitable for drug delivery systems, given their large surface area and ability to transport a bioactive compound. To investigatethe PLLA electrospun membrane releasing properties and mechanism, caffeine was used as the bioactive compound to be tested in the delivery systems.

Caffeine was chosen due to its great solubility in aqueous solution (physiological serum) and because it is probably the pharmacologically active substance most used in the world [24]. Caffeine is most commonly used to improve mental alertness and reduce fatigue, leading to better performance especially in simple psychomotor tasks demanding fast reactions [25]. In combination with painkillers (such as aspirin and acetaminophen) and a chemical called ergotamine it is used for treating migraine headaches. Some people use caffeine for asthma, gallbladder disease, attention deficit-hyperactivity disorder (ADHD) and low blood pressure. Subjective effects of caffeine also include increased feelings of wellbeing, mental energy, motivation, and self-confidence [26].

To understand the releasing mechanism of caffeine through PLLA electrospun membrane, mathematical modeling will be considered through kinetics tests of the delivery systems.

\section{EXPERIMENTAL SECTION}

\subsection{Materials}

The materials mainly used were poly(L-lactic acid) (PLLA), supplied by VELOX GmbH and extra 
pure dichloromethane $\left(\mathrm{CH}_{2} \mathrm{Cl}_{2}, M=84.93 \mathrm{~g} / \mathrm{mol}\right)$, supplied by Scharlau.

\subsection{Methods}

\subsubsection{Polymeric Solution Preparation}

The polymeric solution used to produce the polymeric membrane was obtained by dissolving poly(L-lactic acid) in dichloromethane under constant magnetic agitation. The magnetic agitation remained constant until the PLLA was completely dissolved, which was indicated by the solution becoming translucent and when no solid particles were detected. Complete dissolution was achieved after 2 hours of agitation.

\subsubsection{Electrospinning Technique Used to Produce Membranes}

The process was carried out in an electrospinning prototypal device, developed by INTEXTER; intellectual property rights pertaining to the Polytechnic University of Catalonia.

To carry out the experiment a high voltage power supply, a spinneret (a capillary tube with very small diameter) and a grounded metallic collector plate were required. This technique used a high voltage source to inject a charge of certain polarity into a polymer solution that was, then, accelerated towards a collector of opposite polarity.

\subsubsection{Introduction of Drug into the Membrane}

Caffeine was introduced into the membrane in a second stage, after the electrospinning process, when the membrane was already dried and solidified. The introduction of the drug was carried out in dried medium, with a controlled amount in analytical balance, Mettler Toledo, AG204 model. After placing the drug on the membrane surface, a second layer of membrane was electrospun to recover the drug [27].

\subsubsection{Assessment of Drug Delivery Capabilities}

The polymeric membrane capability of drug delivery was measured through kinetics of drug release into a fluid phase.

Drug release kinetics was carried out in batch methods for membranes with different operational conditions, such as: membranes obtained after different electrospinning times (5, 10 and 20 minutes) and membranes with different drug amounts $(5,10$ and $15 \mathrm{mg})$.

To perform the experiment, membranes were placed in a metallic tambour system, between concentric rings, in order to ensure uniform mass transference along the surface of the membrane from the solid phase to the fluid phase, and also to avoid bending stress. After adjusting the membranes in the metallic tambour system, they were placed in a covered container with a controlled volume of physiological serum (physiological serum solution for intravenous via perforation) for the fluid phase.

The containers prepared for analysis were kept in a bath with a constant temperature of $37^{\circ} \mathrm{C}$. Samples were taken for analysis at regular time intervals, and the drug concentrations released into the fluid phase were determined through spectrophotometric techniques in a UV-vis spectrophotometer, Shimadzu, UV-2401 PC model.

An analysis of each sample and its respective time interval permits design of the kinetics curve (concentration versus time) and, consequently, the determination of release equilibrium time. Equilibrium is reached when the drug concentration value in the fluid phase remains constant with time or when the total amount of drug is released.

\subsubsection{Controlled Drug Release Mathematical Modeling}

The results from the kinetics tests of the delivery systems were used for mathematical modeling of the drug release for a better understanding of the rate-controlling mechanisms, such as diffusion, swelling and erosion of the electrospun polymer.

A semi-empirical equation to describe drug release from polymeric systems, the so-called power law, was used [28]: 


$$
\frac{M_{t}}{M_{\infty}}=k t^{n}
$$

where $M_{t}$ and $M_{\infty}$ are the absolute cumulative amount of drug released at time $t$ and infinite time, respectively; $k$ is a constant incorporation structural and geometric characteristics of the device, and $n$ is the release exponent, indicative of the mechanism of drug release. Through the plot $\log M_{t} / M_{\infty}$ versus $\log t$, the value of $n$ is taken from the slope of the equation straight line and $k$ is taken from $Y$ interception.

Equation (1) has two distinct physical realistic meanings in the two special cases of $n=0.5$ (indicating diffusion-controlled drug release) and $n=1.0$ (indicating swelling-controlled drug release) [28]. Table 1 shows the exponent $n$ of power law and drug release mechanism from polymeric controlled delivery systems [28].

The special case where $n=0.5$, represents the exact solution of Fick's second law for thin films and it is also described by the Higuchi equation in Equation (2).

$$
\frac{M_{t}}{M_{\infty}}=k_{H} \sqrt{t}
$$

where $M_{t}$ and $M_{\infty}$ are the absolute cumulative amount of drug released at time $t$ and infinite time, respectively; $k_{H}$ is the Higuchi constant, reflecting the design variables of the system. $k_{H}$ represents the coefficient of mass transport and it is taken from the slope of the equation straight line of $\sqrt{t}$ versus $M_{t} / M_{\infty}$. Using the Higuchi constant and Fick's second law of diffusion for thin films of thickness $\delta$, we can find the diffusivity of the drug within the polymeric system as it follows.

$$
\begin{aligned}
& \frac{M_{t}}{M_{\infty}}=4\left(\frac{D t}{\pi \delta^{2}}\right)^{\frac{1}{2}} \\
& \frac{M_{t}}{M_{\infty}}=4\left(\frac{D}{\pi \delta^{2}}\right)^{\frac{1}{2}} \cdot \sqrt{t} \\
& k_{H}=4\left(\frac{D}{\pi \delta^{2}}\right)^{\frac{1}{2}}
\end{aligned}
$$

where $D$ is the diffusivity of the drug within the polymeric system and $\delta$ is the thickness of the polymeric film.

\subsubsection{Scanning Electron Microscopy (SEM)}

The surface morphology and thickness of polymeric membranes were examined by a scanning electron microscope (JEOL/JSM-5610) after the samples were dried.

Table 1. Exponent $\boldsymbol{n}$ of the power law and drug release mechanism from polymeric controlled delivery systems of different geometry.

\begin{tabular}{cccc}
\hline & Exponent, $\boldsymbol{n}$ & Drug release mechanism \\
\hline Thin Film & Cylinder & Sphere & \\
0.5 & 0.45 & 0.43 & FickianDiffusion \\
$0.5<n<1.0$ & $0.45<n<0.89$ & $0.43<n<0.85$ & Anomalous Transport \\
1.0 & 0.89 & 0.85 & Case-II Transport \\
\hline
\end{tabular}




\section{RESULTS AND DISCUSSION}

\subsection{The Electrospinning Process}

Despite the fact that electrospinning is relatively simple to process, there are several processing parameters that can greatly affect fiber formation and structure. Grouped in order of relative impact on the electrospinning process, these parameters are: polymer and solvent used; high voltage applied; polymer flow rate; room temperature and relative humidity; capillary tube diameter and capillary-collector distance. Each parameter was studied individually and after all the parameters were set, the ideal electrospinning behavior was achived, as shown in Figure 1.

The adjustment of all parameters provided production of uniform and smooth polymeric membranes, with regular fiber diameter at nanoscale, as shown in Figure 2.

These electrospun polymeric membranes have potential to be used as drug carriers, since the drug can be easily spread and trapped in the middle of the fibers and through diffusion, it can be released. This application, as drug carrier will be discussed in the next section.

A brief list of all parameters analyzed during electrospinning processes and their possible effects on fiber morphology is presented in Table 2.

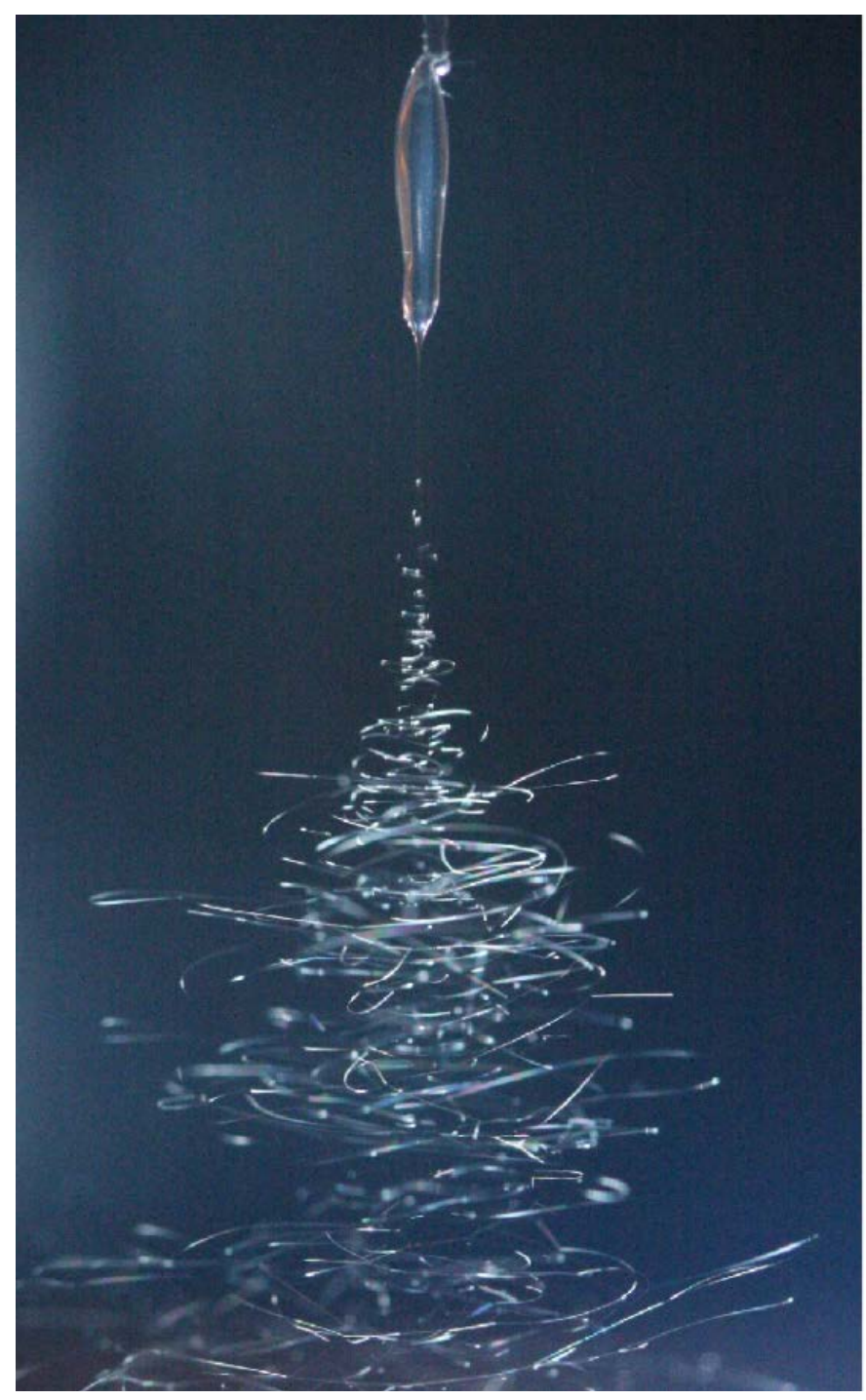

Figure 1. Ideal electrospinning process. 


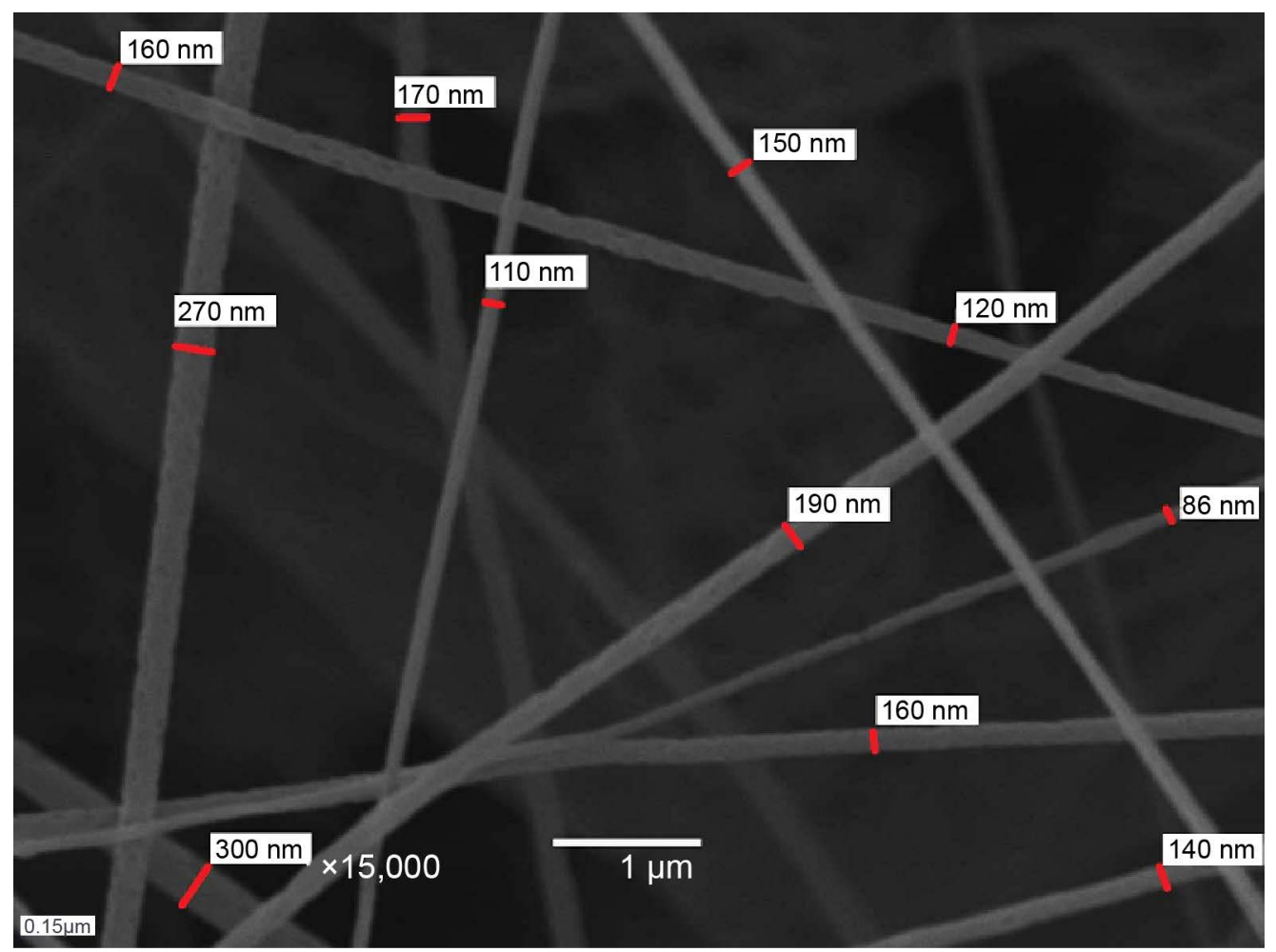

Figure 2. SEM of nanofibers produced by optimized electrospinning process; order of magnitude $15,000 x$.

Table 2. Effect of electrospinning parameters on fiber morphology.

\begin{tabular}{ll}
\hline \multicolumn{1}{c}{ Parameter } & \multicolumn{1}{c}{ Effect on fiber morphology } \\
\hline Solution concentration $\uparrow$ & Fiber diameter $\uparrow$ \\
Solution concentration $\downarrow$ & Difficulties in electrospinning resulting in electrospraying \\
Applied high voltage $\uparrow$ & Fiber diameter $\uparrow$; Bead defect formation; electrospraying \\
Applied high voltage $\downarrow$ & $\begin{array}{l}\text { Defects on fiber structure; capillary tip obstruction due to } \\
\text { premature solvent vaporization }\end{array}$ \\
Flow rate $\uparrow$ & Fiber diameter $\uparrow$; porosity $\uparrow$; bead defect formation \\
Flow rate $\downarrow$ & Capillary tip obstruction due to premature solvent vaporization \\
Temperature $\uparrow$ & Capillary tip obstruction due to premature solvent vaporization \\
Relative humidity $\uparrow$ & Porosity $\uparrow$ \\
$\begin{array}{l}\text { Distance between capillary tip } \\
\text { and collector } \uparrow\end{array}$ & Unstable electric field; process difficulties \\
$\begin{array}{l}\text { Distance between capillary tip } \\
\text { and collector } \downarrow\end{array}$ & Bead defect formation \\
\hline
\end{tabular}




\subsection{Drug Delivery Kinetics}

To test the delivery behavior of the electrospun membrane studied, caffeine was introduced into the electrospun device and releasing tests were carried out in a physiological medium.

The kinetics curves showing the delivery of caffeine in physiological serum through PLLA membrane obtained after 5, 10 and 20 minutes of electrospinning are presented in Figures 3-5.

The kinetics curves presented in Figure 3 show the fast release of caffeine through PLLA membrane, which occurs, mainly, due to the high solubility of caffeine in aqueous media. In addition, the fast release of caffeine is due to the immediate adsorption of the fluid phase by the polymeric membrane, which takes place in the first stage of the releasing process.

When there is a high rate of drug release in the first stage of the releasing process, the internal structure of the polymeric matrix changes considerably, becoming more porous and less restrictive for the diffusion of any compound.

Figure 3 also shows that the releasing behavior is independent on the concentration of caffeine in the polymeric device, since the three tested membranes with different caffeine amount $(5,10$ and $15 \mathrm{mg})$ achieved a kinetics-equilibrium-state at the same time approximately, that is, after 50 minutes in physiological medium. In this case, the polymeric membrane thickness, which is between 0.0662 and $0.1190 \mathrm{~mm}$ (see Table 3), is not enough to control drug release, which is delivered all at once. This kinetics follows a zero-order reaction; it means it does not depend on the concentration.

When increasing the membrane thickness to values between 0.0927 and $0.1424 \mathrm{~mm}$, which is achieved after 10 minutes of electrospinning (Figure 4), it is possible to see that the releasing behavior remains similar to the previous case ( 5 minutes of electrospinning). Figure 4 shows the fast release of caffeine through the PLLA membrane during the first minutes; however, it is followed by a more stable behavior until equilibrium is reached. The time necessary for total caffeine release increases to 100 minutes,

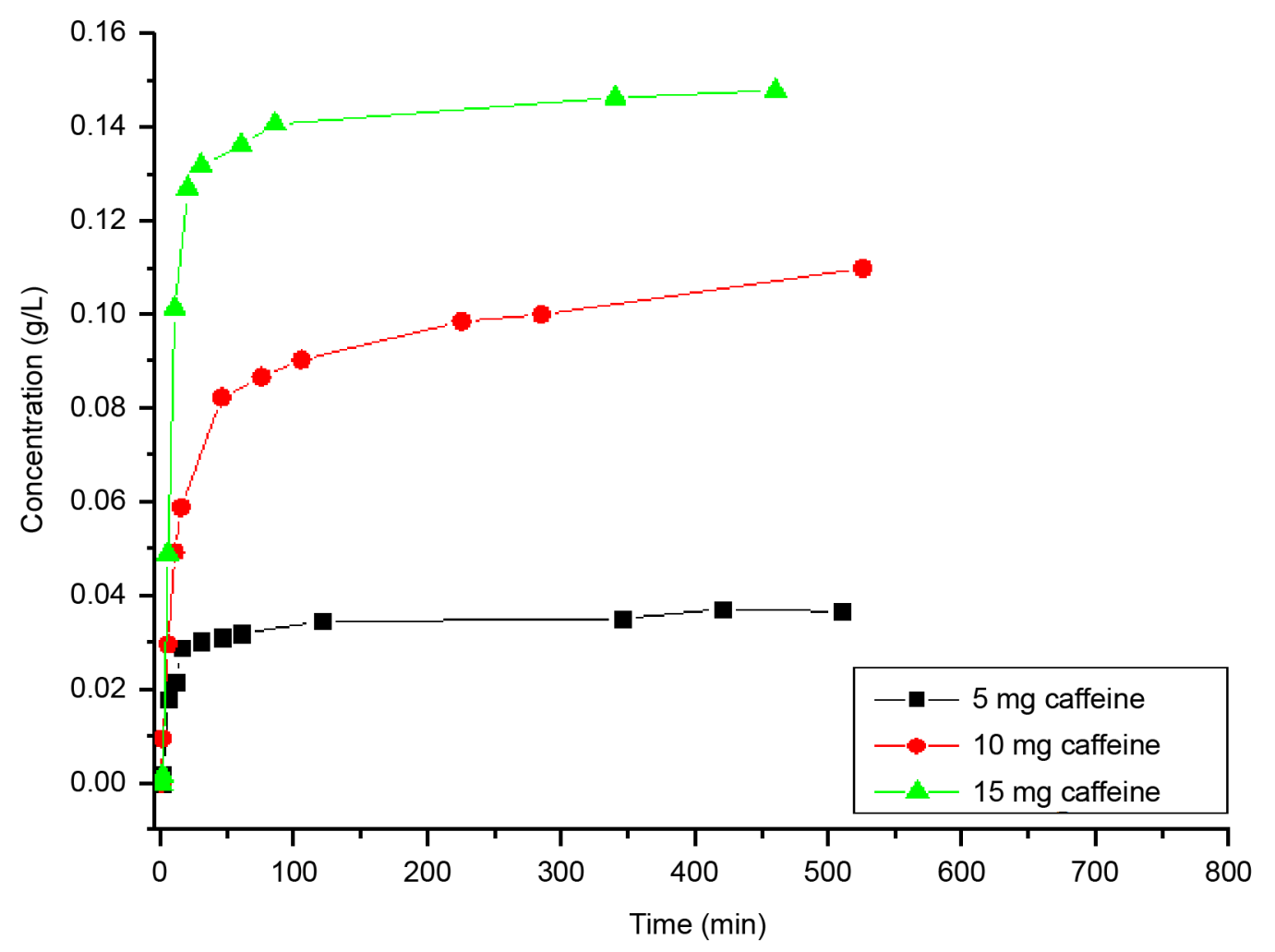

Figure 3. Kinetics of caffeine delivery from PLLA membrane obtained after 5 minutes of electrospinning. 


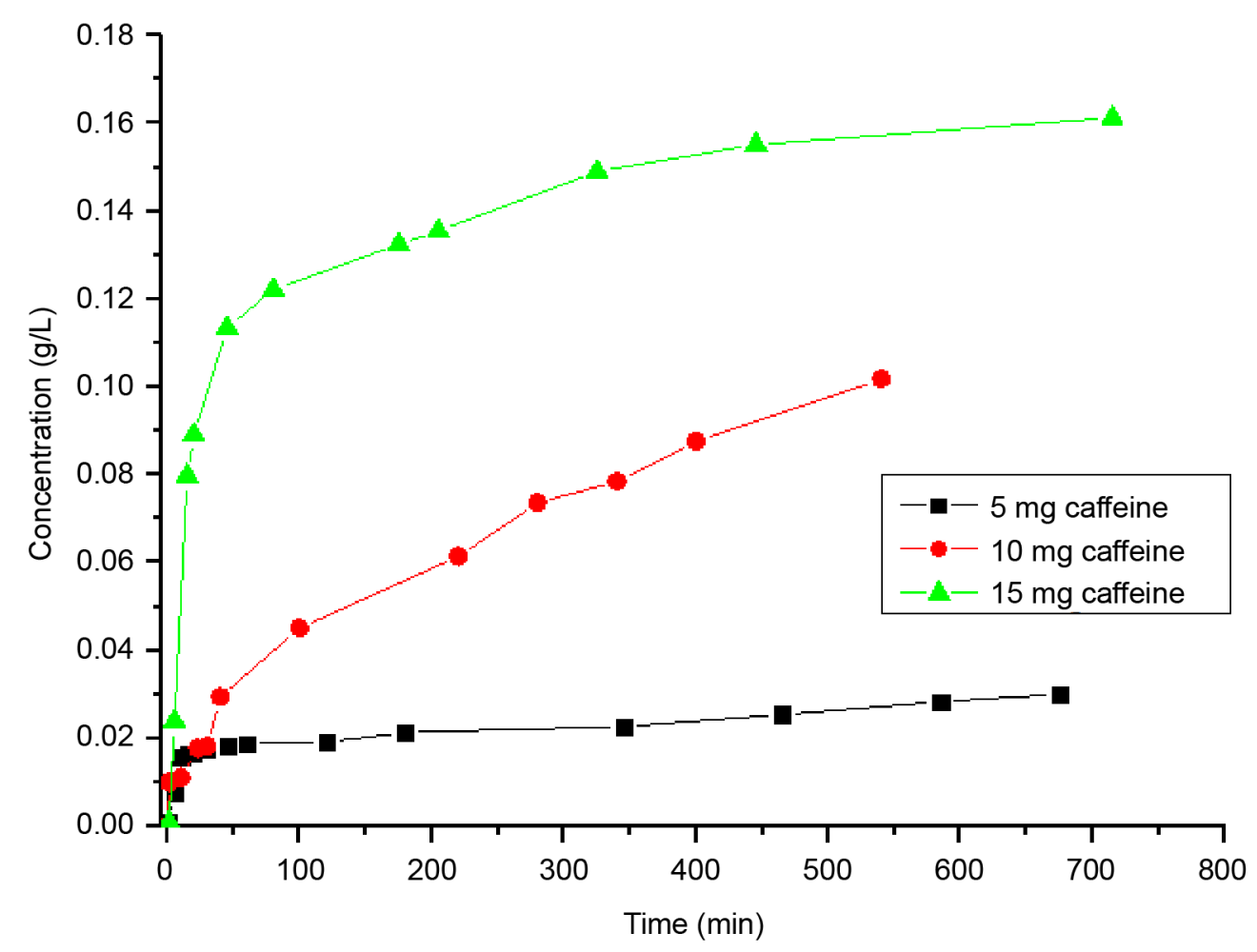

Figure 4. Kinetics of caffeine delivery from PLLA membrane obtained after 10 minutes of electrospinning.

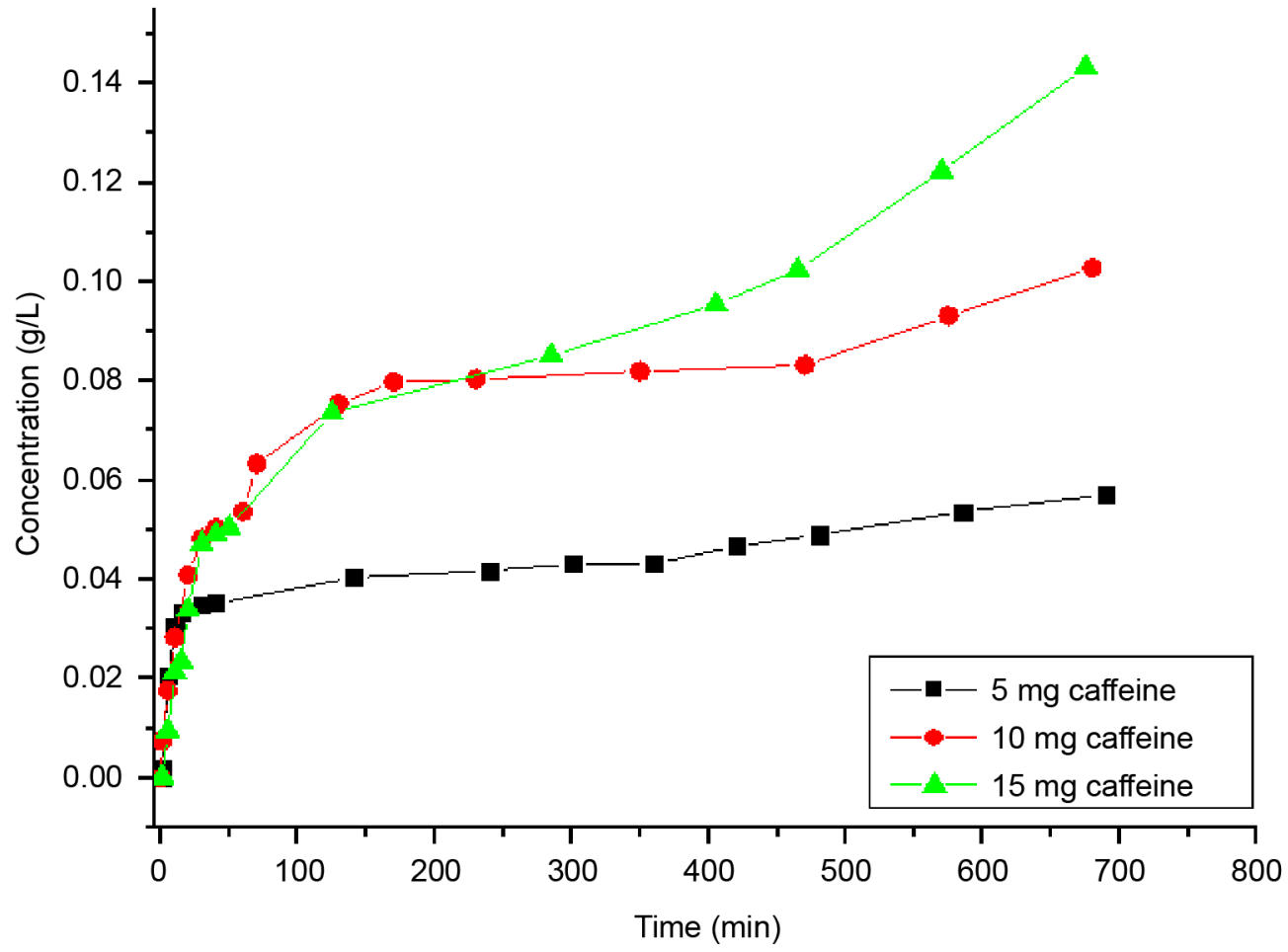

Figure 5. Kinetics of caffeine delivery from PLLA membrane obtained after 20 minutes of electrospinning. 
Table 3. Drug release parameters for PLLA membranes obtained after 5, 10 and 20 minutes of electrospinning.

\begin{tabular}{cccccc}
\hline $\begin{array}{c}\text { Electrospinning } \\
\text { time (min) }\end{array}$ & $\begin{array}{c}\text { Membrane } \\
\text { thickness }(\mathrm{mm})\end{array}$ & $\begin{array}{c}\text { Amount of } \\
\text { caffeine }(\mathrm{mg})\end{array}$ & $\begin{array}{c}\mathbf{n} \text { (Release } \\
\text { mechanism) }\end{array}$ & $\boldsymbol{K}_{H}$ & $\begin{array}{c}\text { Diffusivity } \\
\left(\mathrm{mm}^{2} / \mathrm{min}\right)\end{array}$ \\
\hline 5 & 0.0662 & 5 & 0.1 & 0.17 & $0.621 \mathrm{E}-05$ \\
5 & 0.0926 & 10 & 0.5 & 0.29 & $3.539 \mathrm{E}-05$ \\
5 & 0.1190 & 15 & 0,54 & 1.14 & $9.033 \mathrm{E}-05$ \\
10 & 0.0927 & 5 & 0.52 & 0.18 & $1.366 \mathrm{E}-05$ \\
10 & 0.1130 & 10 & 0.58 & 0.22 & $3.033 \mathrm{E}-05$ \\
10 & 0.1423 & 15 & 0.55 & 0.25 & $6.212 \mathrm{E}-05$ \\
20 & 0.1192 & 5 & 0.54 & 0.22 & $3.375 \mathrm{E}-05$ \\
20 & 0.1424 & 10 & 0.41 & 0.30 & $8.958 \mathrm{E}-05$ \\
20 & 0.1655 & 15 & 0.49 & 0.29 & $11.30 \mathrm{E}-05$ \\
\hline
\end{tabular}

approximately, because the interstitial path to be crossed by the drug has increased with the increasing in membrane thickness. Likewise, the releasing process is independent on caffeine amount, following zero-order kinetics, but dependent on the polymeric membrane thickness.

Using a thicker PLLA membrane, between 0.1192 and $0.1655 \mathrm{~mm}$, obtained after 20 minutes of electrospinning (Figure 5), increases the restriction of caffeine release and equilibrium is reached after 200 minutes, approximately. Here, the addition of extra barrier layers first limits the core hydration process, restraining drug dissolution and diffusion. Then, it also delays drug diffusion, because the drug has a thicker surface to cross.

From the kinetics data, it is possible to find parameters that characterize the releasing process, such as, caffeine releasing mechanism $(n)$ through PLLA membranes. The parameters are presented in Table 3.

Table 3 shows that the releasing mechanism, n, for PLLA membranes obtained after 5 minutes of electrospinning and containing $5 \mathrm{mg}$ of caffeine is exclusively dependent on the polymeric membrane characteristics $(n=1)$, indicating that drug release is controlled by the swelling of the PLLA membrane. According to Siepmann and Peppas, when the exponent $n$ takes a value of 1.0, the drug release rate is independent of time [28]. This case also corresponds to zero-order release kinetics. Here the relaxation process of the macromolecules occurring upon water imbibition into the system is the controlling step.

However, for membranes obtained after 5 minutes of electrospinning and containing 10 and $15 \mathrm{mg}$ of caffeine, as well as the membranes obtained after 10 and 20 minutes of electrospinning, present similar behavior when concerning the releasing mechanism. All membranes exhibit a Fickian diffusion behavior during the drug transport with $n$ equal to 0.5 or approximate values. These membranes, that follow a Fickian behavior during the releasing process, present better control in drug delivery processes, with more stable drug release.

This study presents an understanding of electrospun PLLA drug releasing behavior and through kinetics and mathematical modeling, we could indicate the elements responsible for controlling drug release. However, Higuchi equation could be assumed for all systems in short times, because the release mechanism was governed by Fickian diffusion in all cases $(\mathrm{n}<0.5)$. It was also demonstrated that the membrane thickness is an important factor, acting as a barrier and restraining drug dissolution and diffusion, allowing the drug to be delivered in a controlled manner, and not all at once. 
Although caffeine has been used as the drug test, the results could be extrapolated to any drug with similar solubility in aqueous media, making this delivery device a model for releasing behavior systems, since its high surface to volume ratio, ease of operation, and cost-effectiveness are appealing features for biomedical application.

\section{CONCLUSION}

As has been demonstrated from the experiment results, there were several processing parameters that could greatly affect electrospun fiber formation and structure. The choice of a proper solvent for this process was an important parameter analyzed, because it determined whether fibers were capable of forming, as well as influencing fiber porosity. In this investigation, we demonstrated that electrospun PLLA membranes could be used as drug delivery devices, since PLLA membranes exhibited a Fickian diffusion behavior during the drug transport, presenting better control in drug delivery processes, with more stable drug release. Membrane thickness was also considered an important factor in controlled drug release, restraining drug diffusion, allowing the drug to be uniformly delivered. In conclusion, PLLA/caffeine delivery system was indicated as a model for the releasing behavior of water-soluble drugs, since its high surface to volume ratio, ease of operation, and cost-effectiveness are appealing features for biomedical application.

\section{ACKNOWLEDGEMENTS}

The authors are grateful to CAPES for financial support.

\section{REFERENCES}

1. Loos, M. (2015) Carbon Nanotube Reinforced Composites. CNR Polymer Science and Technology Elsevier, Oxford, 304.

2. Huang, Z.M., Zhang, Y.Z., Kotaki, M. and Ramakrishna, S. (2003) A Review on Polymer Nanofibers by Electrospinning and Their Applications in Nanocomposites. Composites Science and Technology, 63, 2223-2253. https://doi.org/10.1016/S0266-3538(03)00178-7

3. Chilkoti, A., McDaniel, J.R. and Callahan, D.J. (2010) Drug Delivery to Solid Tumors by Elastin-Like Polypeptides. Advanced Drug Delivery Reviews, 62, 1456-1467. https://doi.org/10.1016/j.addr.2010.05.004

4. Li, F., Zhu, Y. and Wang, Y. (2014) Dual-Responsive Drug Delivery System with Real Time Tunable Release Behavior. Microporous and Mesoporous Materials, 200, 46-51. https://doi.org/10.1016/j.micromeso.2014.07.060

5. Ifuku, S., Ikulta, A., Izawa, H., Morimoto, M. and Saimoto, H. (2014) Control of Mechanical Properties of Chitin Nanofiber Film Using Glycerol without Losing Its Characteristics. Carbohydrate Polymers, 101, 714-717. https://doi.org/10.1016/j.carbpol.2013.09.076

6. Whitesides, G.M. and Grzybowski, B. (2002) Self-Assembly at All Scales. Science, 295, 2418-2421. https://doi.org/10.1126/science.1070821

7. Youg, Y., Wang, S., Wang, Y., Wang, X., Wang, Q. and Chen, M. (2014) Advances in Self-Assembled Chitosan Nanomaterials for Drug Delivery. Biotechnology Advances, 32, 1301-1316. https://doi.org/10.1016/j.biotechadv.2014.07.007

8. Hu, X., Liu, S., Zhou, G., Huang, Y., Xie, Z. and Jing, X. (2014) Electrospinning of Polymeric Nanofibers for Drug Delivery Applications. Journal of Controlled Release, 185, 12-21. https://doi.org/10.1016/j.jconrel.2014.04.018

9. Rogina, A. (2014) Electrospinning Process: Versatile Preparation Method for Biodegradable and Natural Polymers and Biocomposite Systems Applied in Tissue Engineering and Drug Delivery. Applied Surface Science, 296, 221-230. https://doi.org/10.1016/j.apsusc.2014.01.098 
10. Agrawal, S., Wendorff, J.H. and Grenier, A. Use of Electrospinning Technique for Biomedical Applications. Polymer, 49, 5603.

11. Nandana, B. and Subhas, C.K. (2010) Electrospinning: A Fascinating Fiber Fabrication Technique. Biotechnology Advances, 28, 325-347. https://doi.org/10.1016/j.biotechadv.2010.01.004

12. Peer, P., Stenicka, M., Pavlinek, V., Filip, P., Kuritka, I. and Brus, J. (2014) An Electrorheological Investigation of PVB Solutions in Connection with Their Electrospinning Qualities. Polymer Testing, 39, 115-121. https://doi.org/10.1016/j.polymertesting.2014.07.016

13. Chong, E.J., Phan, T.T., Lim, I.J., Zhang, Y.Z., Bay, B.H. and Ramakrishna, S. (2007) Evaluation of Electrospun PCL/Gelatin Nanofibrous Scaffold for Wound Healing and Layered Dermal Reconstitution. Acta Materialia, 3, 321.

14. Li, D. and Xia, Y. (2004) Electrospinning of Nanofibers: Reinventing the Wheel? Advanced Materials, 16, 1151-1170. https://doi.org/10.1002/adma.200400719

15. Stitzel, J., Pawlowski, K., Wnek, G., Simpson, D. and Bowlin, G.J. (2001) Arterial Smooth Muscle Cell Proliferation on a Novel Biomimicking, Biodegradable Vascular Graft Scaffold. Journal of Biomaterials Applications, 16, 22. https://doi.org/10.1106/U2UU-M9QH-Y0BB-5GYL

16. Smith, L.A. and Ma, P.X. (2004) Nano-Fibrous Scaffolds for Tissue Engineering. Colloids and Surfaces B: Biointerfaces, 39, 125-131. https://doi.org/10.1016/j.colsurfb.2003.12.004

17. Ramakrishna, S., Fujihara, K., Teo, W.E., Yong, T., Ma, Z. and Ramaseshan, R. (2006) Electrospun Nanofibers: Solving Global Issues. Materials Today, 9, 40-50. https://doi.org/10.1016/S1369-7021(06)71389-X

18. Wang, B., Wang, Y., Yin, T. and Yu, Q. (2010) Applications of Electrospinning Technique in Drug Delivery. Chemical Engineering Communications, 197, 1315. https://doi.org/10.1080/00986441003625997

19. Chakraborty, S., Liao, I.C., Adler, A. and Leong, K.W. (2009) Electrohydrodynamics: A Facile Technique to Fabricate Drug Delivery Systems. Advanced Drug Delivery Reviews, 61, 1043-1054.

https://doi.org/10.1016/j.addr.2009.07.013

20. Liu, S., Zhou, G., Liu, D., Xie, Z., Huang, Y., Wang, X., Wu, W. and Jing, X. (2013) Inhibition of Orthotopic Secondary Hepatic Carcinoma in Mice by Doxorubicin-Loaded Electrospun Polylactide Nanofibers. Journal of Materials Chemistry B, 1, 101. https://doi.org/10.1039/C2TB00121G

21. Zamani, M., Prabhakaran, M.P. and Ramakrishna, S. (2013) Advances in Drug Delivery via Electrospun and Electrosprayed Nanomaterials. International Journal of Nanomedicine, 8, 2997.

22. Moses, M.A., Brem, H. and Langer, R. (2003) Advancing the Field of Drug Delivery. Cancer Cell, 4, 337-341. https://doi.org/10.1016/S1535-6108(03)00276-9

23. Bajpai, A.K., Shukla, S.K., Bhanu, S. and Kankane, S. (2008) Responsive Polymers in Controlled Drug Delivery. Progress in Polymer Science, 33, 1088-1118. https://doi.org/10.1016/j.progpolymsci.2008.07.005

24. Gaspar, S. and Ramos, F. (2016) Caffeine: Consumption and Health Effects. Encyclopedia of Food and Health, 573.

25. Ullrich, S., Vries, Y.C., Kühn, S., Repantis, D., Dresler, M. and Ohla, K. (2015) Feeling Smart: Effects of Caffeine and Glucose on Cognition, mood and Self-Judgment. Physiology \& Behavior, 151, 629-637. https://doi.org/10.1016/j.physbeh.2015.08.028

26. Jovel, C.A.E. and Mejía, F.E.S. (2017) Caffeine and Headache: Specific Remarks. Neurologia, 32, 394-398

27. Stamatialis, D.F., Papenburg, B.J., Gironés, M., Saiful, S., Bettahalli, S.N.M., Schmitmeier, S. and Wessling, M. (2008) Medical Applications of Membranes: Drug Delivery, Artificial Organs and Tissue Engineering. Journal of Membrane Science, 308, 1-34. https://doi.org/10.1016/j.memsci.2007.09.059 
28. Siepmann, J. and Peppas, N.A. (2001) Modeling of Drug Release from Delivery Systems Based on Hydroxypropyl Methylcellulose (HPMC). Advanced Drug Delivery Reviews, 48, 139-157.

https://doi.org/10.1016/S0169-409X(01)00112-0 